\title{
Fluctuations and reweighting of the quark determinant on large lattices
}

\author{
Martin Lüscher, Filippo Palombi* \\ CERN, Physics Department, CH-1211 Geneva 23, Switzerland \\ E-mail: Martin.Luescherecern.ch, Eilippo.Palombiecern.ch
}

\begin{abstract}
We propose to stabilise HMC simulations of lattice QCD with very light Wilson quarks by splitting the quark determinant into two factors and by treating the factor that includes the contribution of the low modes of the Dirac operator as a reweighting factor. In general, determinant reweighting becomes inefficient on large lattices, because the statistical fluctuations of quark determinants increase exponentially with the lattice volume. Random matrix theory and some numerical studies now suggest that the low-mode contribution to the determinant behaves differently, which allows factorisations to be devised that preserve the efficiency of the simulation on large lattices.
\end{abstract}

The XXVI International Symposium on Lattice Field Theory

July 14 - 19, 2008

Williamsburg, Virginia, USA

\footnotetext{
* Speaker.
} 


\section{Introduction}

Simulations of lattice QCD with very light Wilson quarks are potentially affected by algorithmic instabilities, sampling inefficiencies and ergodicity violations. The issue was studied in some detail in ref. [1] and it was concluded that simulations based on the Hybrid Monte Carlo (HMC) algorithm [2] can be expected to be stable in a range of the lattice parameters and the light-quark masses which includes the large-volume regime of QCD at lattice spacings $a \leq 0.1 \mathrm{fm}$.

The algorithm proposed here avoids the instabilities from the beginning by separating the low modes of the Dirac operator from the rest of the modes and by including only the latter in the HMC algorithm. The low modes are then taken into account by reweighting the generated representative ensemble of fields by the appropriate factor. Similar mode separations were recently considered by Jansen et al. [3] and by Hasenfratz et al. [4], partly with the same motivations and partly for other reasons (see refs. [5]-7] for related earlier work).

Low-mode reweighting tends to reduce the statistical fluctuations of observables that are sensitive to the low modes [7] but will only work out if the reweighting factor itself does not fluctuate too much. From this point of view, reweighting by ratios of quark determinants (such as the ones considered below) does not seem to be particularly promising, because quark determinants scale exponentially with the volume of the lattice. Our aim in this report is to show that the situation is actually more favourable than suspected, the main reason being that the low eigenvalues of the Dirac operator (except perhaps for the few lowest ones) fluctuate by no more than a distance inversely proportional to the lattice volume about their mean values.

\section{Determinant factorisation}

We consider lattice QCD with a doublet of light Wilson quarks and any number of heavier quarks. The (massive) light-quark Wilson-Dirac operator is denoted by $D$ and the associated bare current-quark mass by $m$. On average the spectral gap of the hermitian Dirac operator $\gamma_{5} D$ around the origin is then approximately equal to $Z_{\mathrm{A}} m$, where $Z_{\mathrm{A}}$ is the renormalization constant of the isovector axial current [1]].

Determinant reweighting starts from an exact factorisation

$$
\operatorname{det}\left(D^{\dagger} D\right)=W \operatorname{det}\left(\tilde{D}^{\dagger} \tilde{D}\right)
$$

of the light-quark determinant, where $W$ is the reweighting factor and $\tilde{D}$ a modified Wilson-Dirac operator whose determinant is included in the HMC algorithm. In the following, two choices $\tilde{D}_{l}$, $l=1,2$, of the modified operator will be considered for which the associated reweighting factors are of the form

$$
W_{l}=\operatorname{det}\left\{w_{l}\left(D^{\dagger} D\right)\right\}
$$

(see Table 1). Note that the (complex) spectrum of $\gamma_{5} \tilde{D}_{l}$ is in both cases rigorously separated from the origin by a distance of order $\mu$, for all quark masses $m$, very much as in twisted-mass QCD with twisted mass $\mu[8]$. The modified quark determinant $\operatorname{det}\left(\tilde{D}_{1}^{\dagger} \tilde{D}_{1}\right)$ in fact coincides with the quark determinant in twisted-mass QCD.

The inclusion of the modified quark determinant in the HMC algorithm is not expected to give rise to instabilities since the modified Wilson-Dirac operator is safe from having near-zero 


\begin{tabular}{cccc}
$l$ & $\tilde{D}_{l}$ & $w_{l}\left(v^{2}\right)$ & $\left.w_{l}\left(v^{2}\right)\right|_{v^{2} \gg \mu^{2}}$ \\
\hline \hline 1 & $D+i \mu \gamma_{5}$ & $\frac{v^{2}}{v^{2}+\mu^{2}}$ & $1-\frac{\mu^{2}}{v^{2}}+\mathrm{O}\left(v^{-4}\right)$ \\
2 & $\left(D+i \mu \gamma_{5}\right) \frac{\gamma_{5} D-i \mu}{\gamma_{5} D-i \sqrt{2} \mu}$ & $\frac{v^{2}\left(v^{2}+2 \mu^{2}\right)}{\left(v^{2}+\mu^{2}\right)^{2}}$ & $1-\frac{\mu^{4}}{v^{4}}+\mathrm{O}\left(v^{-6}\right)$ \\
\hline \hline
\end{tabular}

Table 1: Modified Dirac operators and associated reweighting factors considered in this report. The mass parameter $\mu>0$ can in principle be set to any value, but good reweighting efficiencies are only achieved if $\mu$ is not much larger than $Z_{\mathrm{A}} m$.

modes. Moreover, we do not foresee any difficulties in applying acceleration techniques such as the Schwarz preconditioning [9] and local deflation [10] to the modified algorithm. In this report, however, the focus will be on the reweighting efficiency and its dependence on the lattice size.

\section{Statistical fluctuations of $W_{l}$}

We now need to distinguish the true QCD expectation value $\langle\mathscr{O}\rangle$ of any observable $\mathscr{O}$ from its expectation value $\langle\mathscr{O}\rangle_{\mathrm{m}}$ in the theory with the modified quark determinant. Only the latter can be estimated directly using the representative ensembles of fields generated by the HMC algorithm, while the first is obtained through

$$
\langle\mathscr{O}\rangle=\frac{\left\langle\mathscr{O} W_{l}\right\rangle_{\mathrm{m}}}{\left\langle W_{l}\right\rangle_{\mathrm{m}}}
$$

Evidently, for the reweighting (3.1) to work out in practice, the statistical fluctuations of $W_{l}$ must be fairly small.

Whether this condition can be met on large lattices is unclear since

$$
W_{l}=\mathrm{e}^{-X_{l}}, \quad X_{l}=\int_{0}^{\mu^{2}} \mathrm{~d} s_{1} \ldots \mathrm{d} s_{l} \operatorname{Tr}\left\{\left(D^{\dagger} D+s_{1}+\ldots+s_{l}\right)^{-l}\right\},
$$

is the exponential of an extensive quantity $X_{l}$. In particular, using the moment-cumulant transformation one can show that

$$
\frac{\left\langle W_{l}^{2}\right\rangle_{\mathrm{m}}}{\left\langle W_{l}\right\rangle_{\mathrm{m}}^{2}}=\left\langle W_{l}\right\rangle\left\langle W_{l}^{-1}\right\rangle=\exp \left\{\sum_{n=1}^{\infty} \frac{2}{(2 n) !}\left\langle X_{l}^{2 n}\right\rangle_{\mathrm{con}}\right\},
$$

where $\left\langle X_{l}^{2 n}\right\rangle_{\text {con }}$ denotes the connected part of $\left\langle X_{l}^{2 n}\right\rangle$. The fluctuations of the reweighting factor thus grow exponentially with the lattice volume $V$ and may therefore rapidly become too large when $V$ is increased. However, as we shall see in the following sections, there are important mechanisms that suppress the fluctuations to the extent that determinant reweighting becomes a viable method in a useful range of parameters.

\section{Suppression of the high modes}

Determinant reweighting is intended for use at quark masses close to or below the range of stability of the HMC algorithm. The values of the renormalized light-quark mass where reweighting 
will be applied are therefore expected to be smaller than $20 \mathrm{MeV}$ or so ${ }^{1}$. At these quark masses, the low end of the spectrum of $\left(D^{\dagger} D\right)^{1 / 2}$ starts at about $Z_{\mathrm{A}} m$ and has an approximately constant density from there up to eigenvalues $\bar{v}_{n, \mathrm{R}}$ of at least $100 \mathrm{MeV}$. In the following, the associated eigenmodes of $D^{\dagger} D$ will be referred to as the "low modes" of the Dirac operator and all other eigenmodes as the "high modes". As explained in the next section, the mass parameter $\mu$ will, in the cases of interest, be less than, say, $2 Z_{\mathrm{A}} m$ and therefore always well below the high eigenvalues of the Dirac operator.

The reweighting factors $W_{l}$ have been chosen so that the eigenvalues $v^{2}$ of $D^{\dagger} D$ larger than $\mu^{2}$ make a monotonically decreasing contribution of order $\mu^{2 l} / v^{2 l}$ to $X_{l}$ (see Table 1). Power counting then shows that the expectation values $\left\langle X_{l}^{2 n}\right\rangle_{\text {con }}$ are ultraviolet convergent except for $\left\langle X_{1}^{2}\right\rangle_{\text {con }}$ which diverges logarithmically. The high modes of the Dirac operator thus contribute a term proportional to $\mu^{4 l} V$ to the fluctuations of $W_{l}$, with a proportionality constant that diverges at most logarithmically in the continuum limit.

On current lattices the product $\mu_{\mathrm{R}}^{4} V$ is usually much smaller than 1 . For lattices of size $2 L^{4}$ with $L \leq 4 \mathrm{fm}$, for example, and if $\mu_{\mathrm{R}} \leq 20 \mathrm{MeV}$, one obtains $\mu_{\mathrm{R}}^{4} V \leq 0.054$. The high-mode contribution to the statistical fluctuations of the reweighting factors $W_{l}$ thus tends to be strongly suppressed, particularly so in the case of $W_{2}$, where there is a second suppression factor proportional to $\mu_{\mathrm{R}}^{4}$.

\section{Fluctuations of the low eigenvalues}

It is still not excluded, however, that the reweighting factors $W_{l}$ receive wildly fluctuating contributions from the low modes of the Dirac operator. There is no obvious suppression mechanism in this case, and since the number of low modes grows proportionally to $V$, it seems likely that determinant reweighting will, in practice, be limited to small lattices.

In order to get some insight into the problem, we worked out the leading term $\left\langle X_{l}^{2}\right\rangle_{\text {con }}$ of the cumulant expansion (3.3) in the standard two-flavour chiral random matrix theory [11]. In this theory, $\left\langle X_{l}^{2}\right\rangle_{\text {con }}$ can be expressed through the spectral density of the Dirac operator and the spectral 2-point correlation function, both of which are known analytically [11, 12]. An integration over the spectral parameters is then still required, but since the integrands are non-singular, it is straightforward to evaluate the integrals numerically.

In random matrix theory, $\left\langle X_{l}^{2}\right\rangle_{\text {con }}$ is a well-defined function of the dimensionless combinations $m \Sigma V$ and $\mu \Sigma V$, where $\Sigma$ denotes the quark condensate in the chiral limit. To a very good approximation, we however found that $\left\langle X_{l}^{2}\right\rangle_{\text {con }}$ only depends on the ratio of these parameters (see Figure 1). Random matrix theory thus suggests that the contribution of the low modes to the fluctuations of the reweighting factors does not change significantly with the volume $V$. Moreover, as can be seen from Figure 1, the contribution is actually quite small up to values of $\mu / m$ equal to 2 or so (note that $Z_{\mathrm{A}}=1$ in random matrix theory and $\mu / m$ therefore corresponds to $\mu_{\mathrm{R}} / m_{\mathrm{R}}$ in lattice QCD).

The outcome of our calculations in random matrix theory can be explained by noting that random matrices have a fairly rigid spectrum, i.e. with high probability, the low eigenvalues are practically unchanged from one random matrix to another. The rigidity of the spectrum may well

\footnotetext{
${ }^{1}$ The renormalized masses are $m_{\mathrm{R}}=Z_{\mathrm{A}} m / Z_{\mathrm{P}}$ and $\mu_{\mathrm{R}}=\mu / Z_{\mathrm{P}}$, where $Z_{\mathrm{P}}$ denotes the renormalization constant of the isovector pseudo-scalar density. The median $\bar{v}_{n}$ of the distribution of the $n$ 'th eigenvalue $v_{n}$ of $\left(D^{\dagger} D\right)^{1 / 2}$ is similarly renormalized through $\bar{v}_{n, \mathrm{R}}=\bar{v}_{n} / Z_{\mathrm{P}}$. Values of these quantities quoted in physical units refer to the $\overline{\mathrm{MS}}$ renormalization scheme at $2 \mathrm{GeV}$.
} 

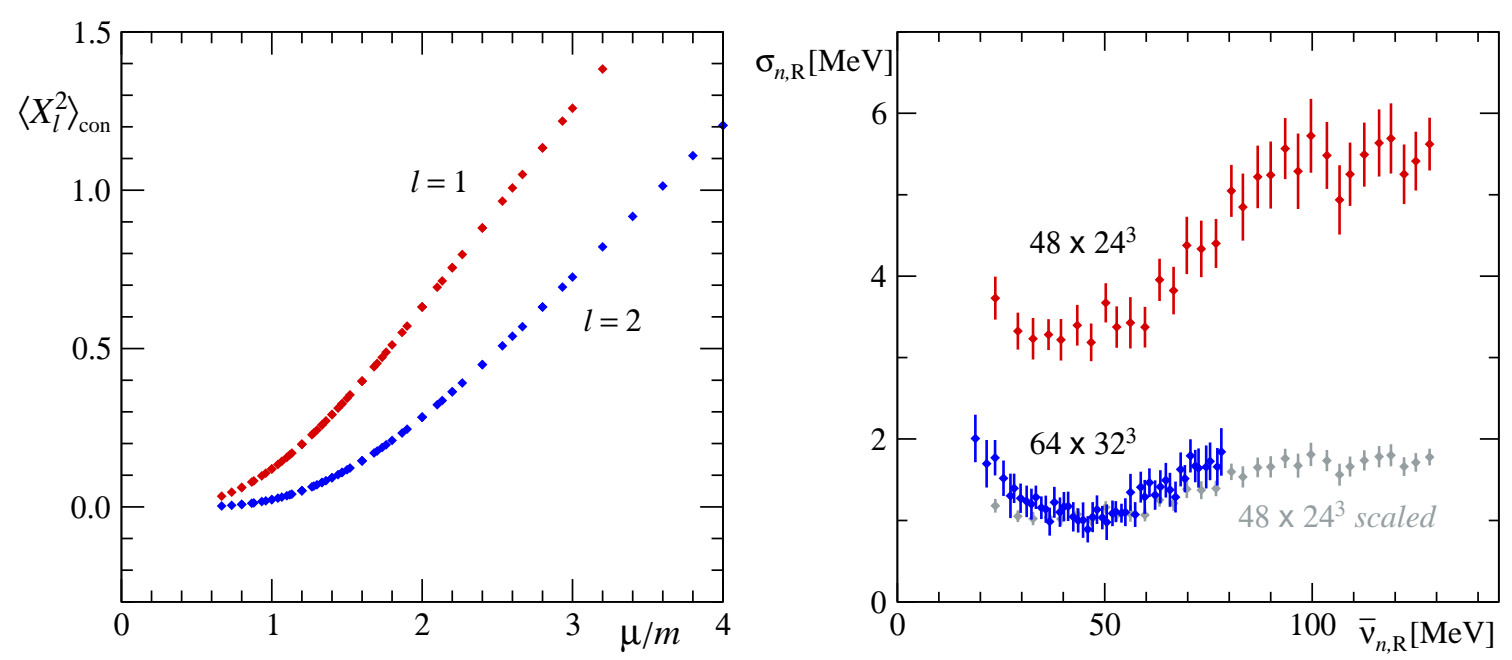

Figure 1: Values of $\left\langle X_{l}^{2}\right\rangle_{\text {con }}$ computed in random matrix theory at $m=5, \ldots, 30 \mathrm{MeV}$ and $\mu=20, \ldots, 44$ $\mathrm{MeV}$, assuming $\Sigma=(250 \mathrm{MeV})^{3}$ and $V=(4.5 \mathrm{fm})^{4}$ (plot on the left). The plot on the right shows the widths of the distributions of the first 32 (48) eigenvalues of $\left(D^{\dagger} D\right)^{1 / 2}$ on a lattice of size $48 \times 24^{3}\left(64 \times 32^{3}\right)$. In both cases the lattice spacing and the renormalized sea-quark mass are approximately equal to $0.08 \mathrm{fm}$ and $25 \mathrm{MeV}$ respectively. The grey points labelled " $48 \times 24^{3}$ scaled" are the $48 \times 24^{3}$ data scaled by the ratio $(24 / 32)^{4}$ of the lattice volumes.

be related to the fact that the Vandermonde determinant, which appears in the joint distribution of the eigenvalues, gives rise to a repulsive force between neighbouring eigenvalues. In any case, since the fluctuations of the low eigenvalues are of order $(\Sigma V)^{-1}$ and since there are $\mathrm{O}(V)$ eigenvalues, their contribution to the fluctuations of the reweighting factor $W_{l}$ will obviously remain bounded at large $V$.

In lattice QCD with Wilson quarks, chiral symmetry is not exactly preserved and it is therefore not guaranteed that the eigenvalues of the lattice Dirac operator behave in the same way. Numerical studies of the $\mathrm{O}(a)$-improved two-flavour theory however suggest that the widths of the eigenvalue distributions scale roughly like $1 / V$, as in random matrix theory, except for the widths measured close to the threshold of the spectrum (see Figure 1$)^{2}$. In the Wilson theory, the contribution of the low modes to the fluctuations of the reweighting factor is therefore slowly increasing with the lattice size, an effect that is likely to become smaller as one moves closer to the continuum limit.

\section{Computation of the reweighting factors}

An exact calculation of the reweighting factors $W_{l}$ is normally not possible and actually not required. Stochastic estimators can be used instead, or perhaps some combination of a stochastic estimator and a (not necessarily exact) projector to the few lowest modes of the Dirac operator. Here we only consider the most obvious choice, where a set $\eta_{k}(x), k=1, \ldots, N$, of pseudo-fermion

\footnotetext{
${ }^{2}$ The representative ensembles of gauge-field configurations used for our numerical studies were generated by the authors of ref. [13] and were made available to us through the CLS community effort.
} 
$48 \times 24^{3}$

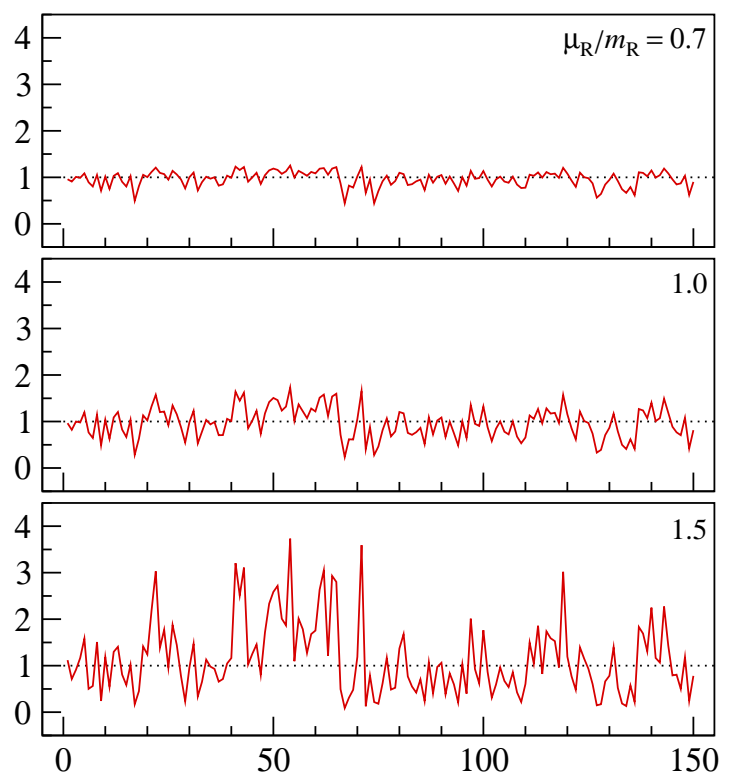

$64 \times 32^{3}$

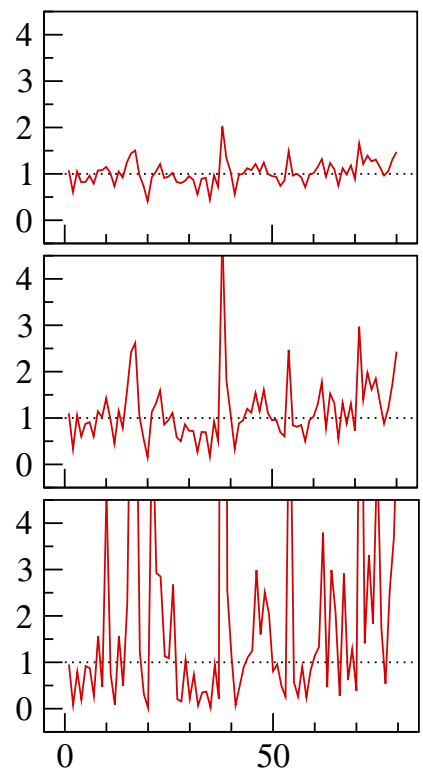

Figure 2: Reweighting factor $W_{2,24}$ normalized by its median at $m_{\mathrm{R}} \simeq 25 \mathrm{MeV}$ and $\mu_{\mathrm{R}} / m_{\mathrm{R}}=0.7,1.0,1.5$ (from top to bottom), calculated for a set of independent gauge-field configurations on a $48 \times 24^{3}$ (plots on the left) and a $64 \times 32^{3}$ lattice (plots on the right) with spacing $a \simeq 0.08 \mathrm{fm}$.

fields with action

$$
S_{\eta}=\sum_{k=1}^{N}\left(\eta_{k}, \eta_{k}\right)
$$

is added to the theory and the reweighting factor $W_{l}$ is replaced by

$$
W_{l, N}=\frac{1}{N} \sum_{k=1}^{N} \exp \left\{\left(\eta_{k},\left[1-w_{l}\left(D^{\dagger} D\right)^{-1}\right] \eta_{k}\right)\right\}
$$

The simulation then proceeds as before and the reweighting factor $W_{l, N}$ is calculated according to eq. (6.2), using, for each gauge field, $N$ randomly chosen pseudo-fermion fields. This procedure is correct for any $N \geq 1$, but it pays to set $N$ to values significantly larger than 1 , because the variance of $W_{l, N}$ decreases when $N$ is increased (and eventually converges to the variance of $W_{l}$ ).

For illustration the Monte Carlo time series of $W_{2,24}$ calculated on the lattices previously considered are plotted in Figure 2. In all these cases, little would be gained by choosing more pseudofermion fields or by separating the lowest modes of the Dirac operator (such a mode separation may, however, be required at smaller quark masses).

Figure 2 also shows that the fluctuations of $W_{2,24}$ increase with the lattice size and that they are quite sensitive to the value of $\mu_{\mathrm{R}} / m_{\mathrm{R}}$. In particular, by decreasing the latter, the fluctuations are quickly reduced to acceptable levels on both lattices. The fluctuations of $W_{1,24}$ at $\mu_{\mathrm{R}} / m_{\mathrm{R}}=0.7$ and 1.0 are, incidentally, practically the same as those of $W_{2,24}$ at $\mu_{\mathrm{R}} / m_{\mathrm{R}}=1.0$ and 1.5 , respectively. On the lattices considered and before the performance of the HMC part of the algorithm is determined, it is therefore not clear whether the first or the second factorisation of the quark determinant is preferable. 


\section{Conclusions}

In this report we showed that determinant reweighting is likely to work out in lattice QCD if a factorisation of the quark determinant is chosen where the high-mode contribution to the reweighting factor is sufficiently suppressed. Somewhat surprisingly to us, the statistical fluctuations of the reweighting factor then do not grow rapidly with the volume of the lattice, a property that can be traced back to the rigidity of the spectrum of the low eigenvalues of the Dirac operator.

We are now quite confident that simulations of the Wilson theory at very small quark masses can be stabilised in this way, but still need to prove this by actually performing such simulations using the proposed determinant factorisations.

\section{References}

[1] L. Del Debbio, L. Giusti, M. Lüscher, R. Petronzio, N. Tantalo, Stability of lattice QCD simulations and the thermodynamic limit, JHEP 0602 (2006) 011 [arXiv: hep-lat/0512021v1]

[2] S. Duane, A. D. Kennedy, B. J. Pendleton, D. Roweth, Hybrid Monte Carlo, Phys. Lett. B195 (1987) 216

[3] K. Jansen, A. Nube, A. Shindler, C. Urbach, U. Wenger, Exploring the epsilon regime with twisted mass fermions, PoS (LATTICE2007) 084 arXiv:0711.1871v1]

[4] A. Hasenfratz, R. Hoffmann, S. Schaefer, Reweighting towards the chiral limit, Phys. Rev. D78 (2008) 014515 arXiv:0805.2369v1; Low energy chiral constants from epsilon-regime simulations with improved Wilson fermions, Phys. Rev. D78 (2008) 054511 [arXiv:0806.4586v2]

[5] A. Duncan, E. Eichten, H. Thacker, An Efficient algorithm for QCD with light dynamical quarks, Phys. Rev. D59 (1999) 014505 arXiv:hep-lat/9806020v1

[6] A. Duncan, E. Eichten, Y. Yoo, Unquenched QCD with light quarks, Phys. Rev. D68 (2003) 054505 arXiv:hep-lat/0209123v1]

[7] M. Della Morte, R. Hoffmann, F. Knechtli, U. Wolff (ALPHA collab.), Impact of large cutoff-effects on algorithms for improved Wilson fermions, Comput. Phys. Commun. 165 (2005) 49 [arXiv:hep-lat/0405017v2]

[8] R. Frezzotti, P. A. Grassi, S. Sint, P. Weisz (ALPHA collab.), Lattice QCD with a chirally twisted mass term, JHEP 0108 (2001) 058 [arXiv: hep-lat/0101001v2]

[9] M. Lüscher, Schwarz-preconditioned HMC algorithm for two-flavour lattice QCD, Comput. Phys. Commun. 165 (2005) 199 [arXiv:hep-lat/0409106v1]

[10] M. Lüscher, Local coherence and deflation of the low quark modes in lattice QCD, JHEP 0707 (2007) 081 arXiv: $0706.2298 \mathrm{v}$ ]; Deflation acceleration of lattice QCD simulations, JHEP 0712 (2007) 011 arXiv:0710.5417v1]

[11] J. J. M. Verbaarschot, I. Zahed, Spectral density of the QCD Dirac operator near zero virtuality, Phys. Rev. Lett. 70 (1993) 3852 arXiv:hep-th/9303012v1]

[12] P. H. Damgaard, S. M. Nishigaki, Universal spectral correlators and massive Dirac operators, Nucl. Phys. B 518 (1998) 495 arXiv: hep-th/9711023v5

[13] L. Del Debbio, L. Giusti, M. Lüscher, R. Petronzio, N. Tantalo, QCD with light Wilson quarks on fine lattices. II. DD-HMC simulations and data analysis, JHEP 0702 (2007) 082 arXiv:hep-lat/0701009v1] 